\title{
Sistema electrónico braille para la ayuda en el aprendizaje de personas no videntes
}

\author{
Oscar Patricio Loza Peñaloza*
}

\section{Introducción}

Durante muchos años los métodos de enseñanza del sistema de lectoescritura braille han sido transmitidos de una manera rústica y sin llevar técnicas que busquen fomentar el mejoramiento del aprendizaje. Muchas personas y entidades que tienen a su cargo la puesta en marcha de la tecnología han desarrollado herramientas que, de alguna forma, ayudan a las personas con algún tipo de discapacidad pero, lamentablemente, la realizan utilizando los conocimientos que han adquirido durante mucho tiempo y no poniéndose del lado de las verdaderas necesidades de la persona que las va a utilizar.

Braille Electrónico se ha desarrollado conjuntamente con la ayuda de no videntes para darle características de control de fácil uso y varios entornos en donde, además de leer, se pueda practicar la escritura del Sistema Braille.

\section{2. ¿Qué es Braille Electrónico?}

EI "SISTEMA ELECTRONICO BRAILLE PARA AYUDA EN EL APRENDIZAJE DE PERSONAS NO VIDENTES" O "E_BRAILLE" es un dispositivo (software y hardware), de

\footnotetext{
* Ex-alumno de la Universidad Politecnica Salesiana-Cuenca
} 
aporte para la sociedad del conocimiento y de la educación de personas no videntes, puesto que todos los individuos deben tener la posibilidad de acceder a una educación, sin que las personas con discapacidad sean una excepción.

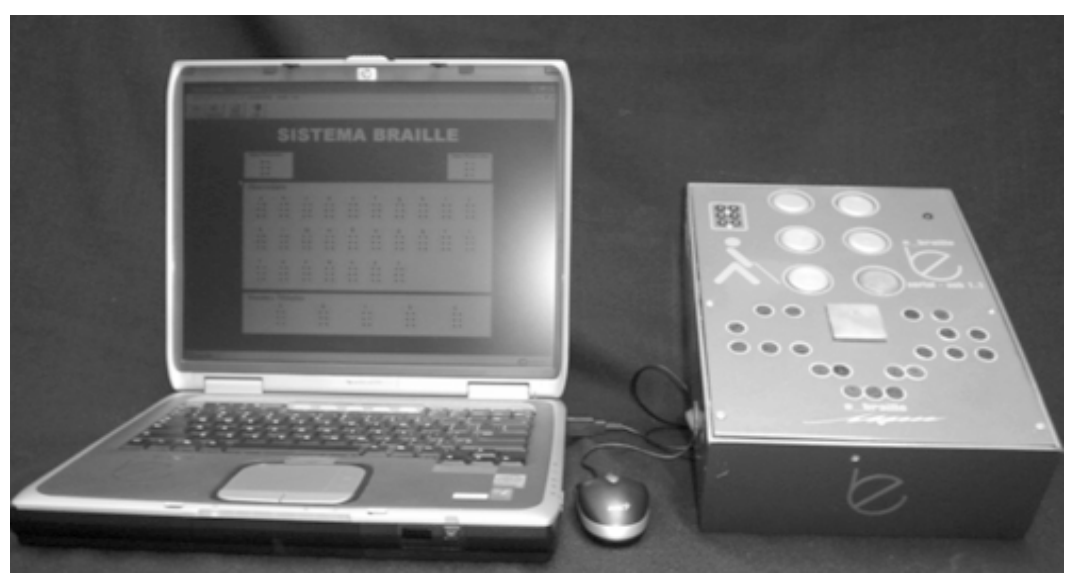

Figura 1. Sistema Braille Electrónico (e_braille).

\section{Un poco de historia ${ }^{1}$}

Durante centenares de años no hubo un sistema de lectura y escritura apropiado para ser empleado en la educación de las personas ciegas y muchos pensaban que estos no eran educables. Por supuesto que lo anterior no es efectivo, el camino hacia la alfabetización de los ciegos puede decirse que empezó en 1786 en París y que la primera piedra la puso Valentín Haüy, un filántropo francés que sintió el impulso de liberar de la mendicidad a los muchos marginados ciegos que se ganaban la vida pidiendo limosna o mal tocando música por las calles de la capital francesa, a finales del siglo XVIII. La ceguera no debía asociarse obligatoriamente a la ignorancia y la dependencia de los demás.

\footnotetext{
${ }^{1}$ CRESPO, Susana. Glosario referido al Sistema Braille, Documento.
} 
Haüy desarrolló, no obstante, el primer método de impresión de libros para ciegos que consistía en presionar una cartulina mojada sobre caracteres de gran tamaño hechos de plomo. Los libros resultantes eran enormes y muy pesados. Cada volumen pesaba alrededor de nueve kilos. Por otra parte, su lectura era excesivamente lenta, debiendo recorrer con la yema del dedo cada carácter hasta reconocerlo y poder seguir con el siguiente de forma que al final de una palabra difícilmente recordaban sus primeras letras. Por otra parte, el método de Valentín Haüy hacía posible la lectura, pero en ningún caso la escritura por parte de los ciegos.

Un avance cualitativamente importante fue la propuesta de Charles Barbier, un militar que había inventado un modo de escritura y lectura basado en puntos y rayas en relieve con el fin de que los soldados pudiesen descifrar por la noche consignas secretas, y lo presentó en el colegio de "La Institución Real para Niños Ciegos de París" donde se encontraba internado, contando pocos más de diez años, Louis Braille, que se había quedado ciego a los tres años. El nuevo método se llamaba sonografía y se basaba en diversas combinaciones de puntos y rayas formando signos que representaban los diferentes sonidos del lenguaje, no las letras del alfabeto. Para escribirlo, se usaba una especie de regla con siete surcos poco profundos y una pinza que se deslizaba verticalmente siguiendo los renglones. En la pinza había una especie de ventanitas donde podían formarse los signos presionando con un punzón sobre la hoja de papel que se colocaba entre la pinza y la regla.

Louis Braille, sobresaliente en inquietudes y capacidad de trabajo, decidió abordar la tarea de simplificar el método sonográfico de Barbier y completarlo en los aspectos que resultarían imprescindibles para disponer de un auténtico alfabeto.

Unos meses más tarde, había encontrado un medio de formar todas las letras, los acentos, los signos de puntua- 
ción y los signos matemáticos utilizando solo seis puntos y algunas rayas horizontales que, más adelante, eliminaría.

El resultado no es solamente una modificación del método de Barbier sino que supone un cambio esencial de su propio planteamiento. El hecho de que Louis Braille sea ciego le permite experimentar personalmente sus investigaciones y esta característica explica la perfecta acomodación de la forma y el tamaño de los signos a la naturaleza del tacto en la yema del dedo para facilitar su rápida identificación en el mínimo tiempo y con el menor movimiento. Al final, después de largas investigaciones, el nuevo sistema de lectoescritura tendría exclusivamente seis puntos, con los que se podrían formar 63 signos diferentes, incluyendo el espacio en blanco.

Y no solo inventa el alfabeto: lo adapta a las matemáticas y a las ciencias, desarrolla un sistema de abreviaturas y lo que resulta más interesante, lo adecuó también para la música. La llamada "musicografía" braille es, realmente, muy inteligente, ya que transforma la escritura musical, de vertical, en otra horizontal y consecutiva.

En el año 1840 se acepta oficialmente el sistema braille y en 1878, un congreso internacional decide promoverlo en el mundo entero, al considerarlo el mejor sistema para el tacto.

Louis Braille, que había nacido el 4 de enero de 1809, murió de tuberculosis, en París, a la edad de 43 años y fue enterrado en Coupvray, su pueblo natal. Hoy día sus restos descansan en el Panteón de Hombres llustres no muy lejos del edificio que aún ocupa el Instituto de Jóvenes Ciegos, donde falleciera el 6 de enero de 1852.

\section{El Sistema Braille ${ }^{2}$}

El braille es un sistema de puntos en relieve que permite a los ciegos leer y escribir al tacto. Todo el sistema deriva de una

${ }^{2}$ VILLAVICENCIO, Daniel. Director de la Sociedad de no videntes del Azuay. Asesoramiento.

212 
distribución de seis puntos conocidos como la celdilla braille. Cada distribución de puntos, como también cada espacio en blanco, ocupa una celdilla. Para ayudar a identificar las posiciones de los puntos, que según su distribución representen los distintos signos del braille, Louis Braille numeró las posiciones de los puntos dentro de su celdilla de arriba hacia abajo 1-2-3 al lado izquierdo y 4-5-6 al lado derecho (Figura 2).

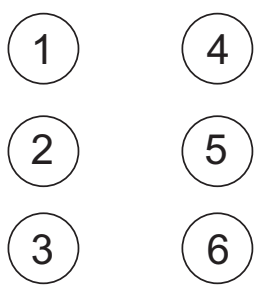

Figura 2. Signo generador.

Al arreglar estos puntos en diferentes posiciones y combinaciones puede llegarse a sesenta y tres signos 0 caracteres de braille distintos.

Todo idioma se vale de acentos o de otros signos. Un lector de braille en inglés, por ejemplo, solo necesita aprender un signo general para "acento" el que, al aprender una letra, indica que hay un acento u otro signo similar en lo impreso y en esa forma descarta la necesidad de aprender los símbolos especiales que emplean los diferentes idiomas. En el español y el francés, sin embargo, ambos idiomas en que todas las vocales pueden ir o no ir acentuadas (cambiando el acento o el distinto tipo de acento, a veces el significado de la palabra), se ha visto la necesidad de tener signos especiales que representen las vocales acentuadas. Otro principio que posibilita la utilización máxima de las 63 combinaciones de puntos es la asignación de valores en relación con la notación específica empleada en una cierta materia o idioma. Así, el octavo signo de la tercera línea representa la letra "w" para el lector de braille, siempre que 
esté leyendo en inglés, alemán u otro idioma que haga uso de esta letra.

Existen tres niveles dentro del Sistema Braille. El primer nivel, denominado Braille integral y otros dos niveles, que incluyen distintos tipos de abreviaciones y reducciones en las palabras y conforman la estenografía Braille. La estenografía Braille es similar a nuestra taquigrafía, pero posee signos propios.

1. El primer nivel, mantiene una relación de uno a uno con las letras del alfabeto, es decir, que a cada una de las letras le corresponde un determinado signo.

2. El segundo nivel, es el inicio de la estenografía e incluye las palabras abreviadas con un solo signo. Por ejemplo, la palabra "que" se estenografía con la "q".

3. El tercer nivel, incluye todas las contracciones y las palabras abreviadas con dos o más signos. Por ejemplo, la palabra "ciego" se estenografía con la "c" y la " $g$ ".

Las 63 combinaciones representan las letras del alfabeto, los números, los signos matemáticos, las letras extranjeras, etc., es decir, se utilizan las mismas configuraciones para signos diferentes. Esto implica que la persona que lee y escribe, por medio de este código, debe estar muy atenta para utilizar o anteponer distintos signos.

Además, debemos sumarle otra complejidad: el cambio de lugar de uno o más puntos transforma una letra en otra, lo cual supone que cambie la palabra o sea incomprensible.

El Sistema Braille se escribe de derecha a izquierda si se realiza con pizarra y punzón y de izquierda a derecha si se escribe a máquina. Esta última sería la manera ideal para iniciar a cualquier niño en la escritura, pero su fabricación extranjera y el consiguiente valor en dólares la torna imposible de poseer para más de la mitad de los niños y adultos de nuestro país. 
La lectura se realiza de izquierda a derecha, con ambas manos si se utiliza una técnica correcta y con las yemas de los dedos mayor e índice. Tanto la escritura aun cuando se utilice la estenografía como la lectura por el carácter analítico del tacto, se realizan de manera más lenta; por lo tanto, la persona ciega siempre requiere de una mayor cantidad de tiempo para su realización. No obstante, se puede lograr un nivel adecuado a partir de la motivación personal y el ejercicio.

\section{Descripción del proyecto}

Es una realidad que una persona con discapacidad está limitada al momento de realizar ciertos tipos de tareas y en nuestro caso esta claro que un no vidente no puede saber qué es lo que esta escrito en un computador por ello el desarrollo de esta investigación esta en realizar un programa que interprete el texto editado para que pueda ser enviado por el puerto serial o USB de un computador y ser analizado por un módulo electrónico que maneje dispositivos mecánicos y así representar el alfabeto braille acorde al texto inicial. (Figura 3).

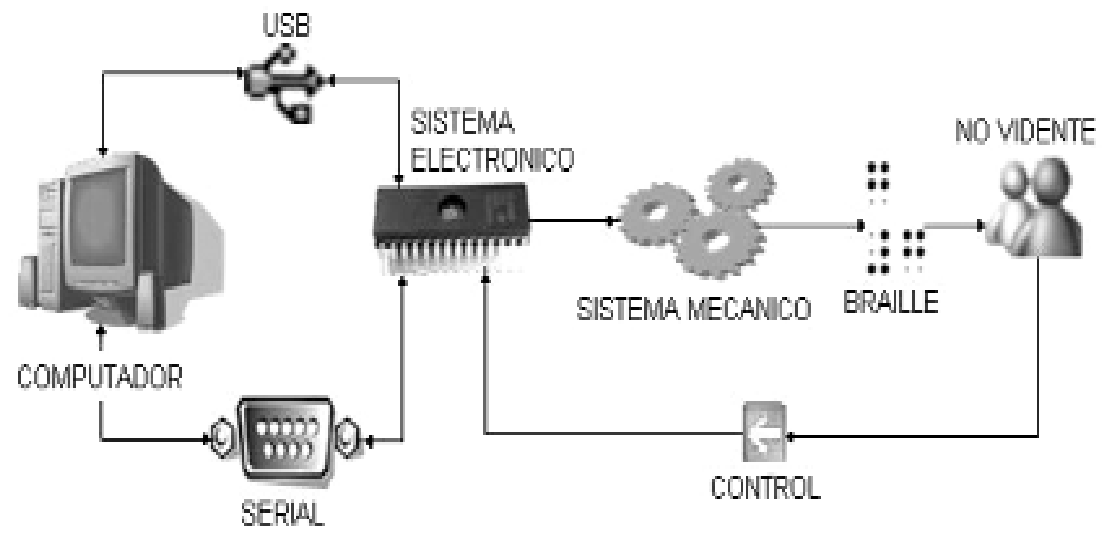

Figura 3. Diagrama general de diseño. 
La necesidad de implementar un sistema capaz de interactuar entre un computador y una persona con discapacidad visual, nos ha llevado a plantear la siguiente hipótesis:

"El procesamiento de datos mediante un sistema electrónico y la ayuda de un sistema mecánico, permitirá a una persona no-vidente a interpretar texto editado en un computador basado en el sistema de lectura Braille".

El alcance social que ostenta este proyecto está en incrementar la facilidad de aprendizaje de las personas discapacitadas mediante el desarrollo de una tecnología desde una perspectiva globalizada e integradora, abarcando tan diversas e importantes áreas como la educación, tiempo libre, el trabajo, la familia, el control del entorno y todo ello como medio facilitador de mayores cotas en la calidad de vida de estas personas.

Además esta en el beneficio para instituciones que utilizarían este proyecto para la enseñanza de este sistema de lectura de una manera más eficaz y eficiente.

Otro alcance importante está en incrementar la interacción de las personas discapacitadas con el manejo de un computador.

El software del presente proyecto esta dividido en dos partes:

Software del computador

La creación del programa del Braille Electrónico bajo Visual Basic ha conducido al estudio de las necesidades que han dado origen a la creación de Braille Electrónico. Es lo que se llama análisis de la aplicación. Es la primera fase que debe tener siempre un programa y es también la más olvidada entre los programadores. Una aplicación no se inicia con el teclado, sino sobre un papel.

La interfaz será la principal vía de comunicación hombre-máquina, tanto para salida de datos como para entrada. Será necesario partir de una o varias ventanas (for- 
mularios) a las que les irán añadiendo los controles necesarios.

Los formularios y controles de Visual Basic son objetos que exponen sus propios métodos, propiedades y eventos. Las propiedades se pueden considerar como atributos de un objeto, los métodos como sus acciones y los eventos como sus respuestas.

El programa del Braille Electrónico utiliza la interfaz de documentos múltiples (MDI) ya que nos permite crear una aplicación que mantiene varios formularios dentro de un único formulario contenedor. Es decir, se tiene una interfaz principal el cual contiene a ventanas secundarias. Se presenta en la figura 4 un diagrama lógico general de cómo esta estructurado la interfaz del programa del Braille Electrónico.

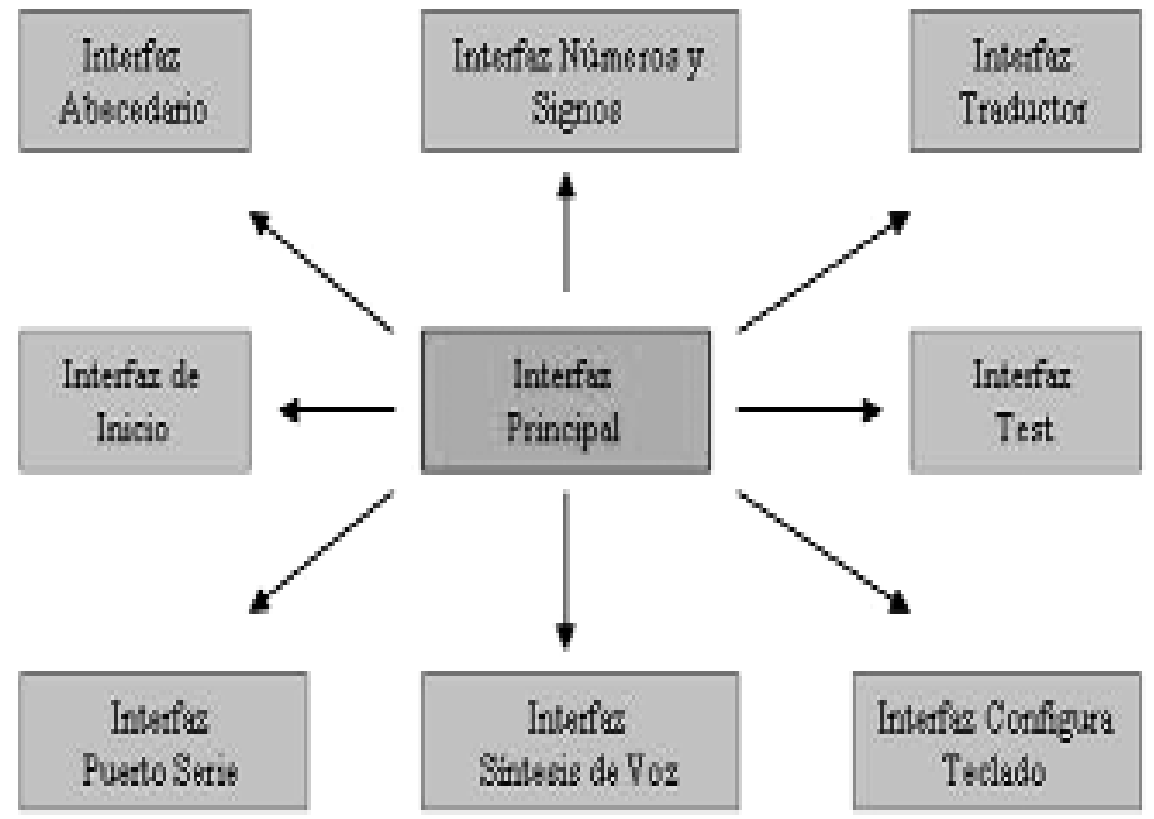

Figura 4. Estructura del Interfaz del programa del Braille Electrónico. 
Una parte importante del software que vale la pena mencionar es un control ActiveX diseñado para la conversión de una entrada escrita en palabras, a una salida pronunciada, simulando el proceso humano de leer en voz alta utilizando los sistemas de síntesis de voz.

Los sistemas de síntesis de voz, son aquellos que convierten una entrada escrita en palabras, a una salida pronunciada, simulando el proceso humano de leer en voz alta. Estos sistemas son también conocidos como sistemas de texto a voz (TTS, siglas de las palabras en inglés TextTo-Speech). Para su aplicación se ha seleccionado Microsoft Text to speech, por su compatibilidad con el lenguaje de programación Visual Basic y su empleo no requiere la compra de licencia. En la figura 5 se describe el funcionamiento del sistema TTS mediante un diagrama.

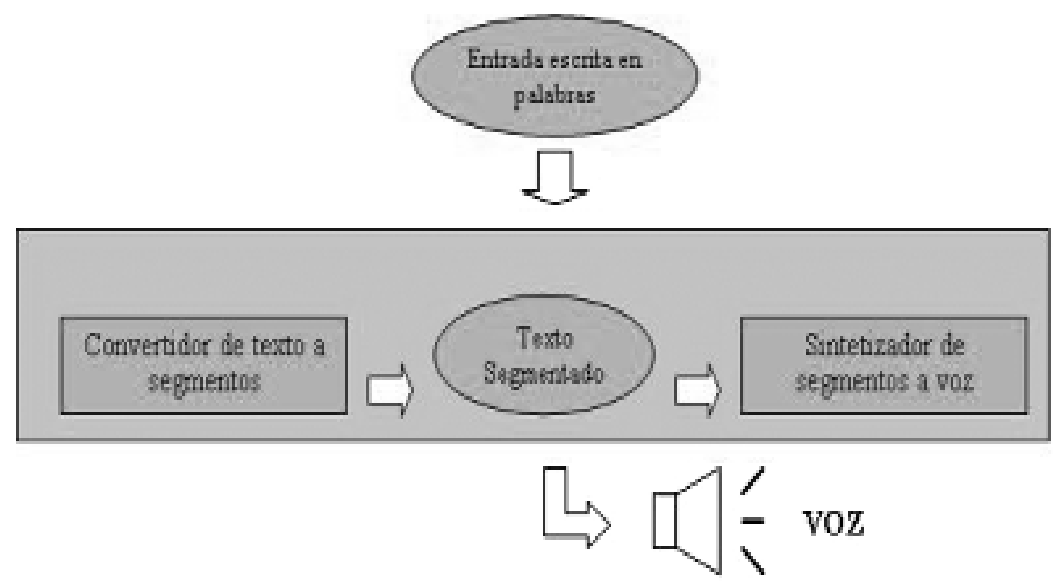

Figura 5. Estructura de un Sistema TTS.

- Software del microcontrolador

Una parte del software del microcontrolador implementa todas las instrucciones para la configuración del dispositivo, el envío, la recepción de datos el manejo de puertos. Primeramente se configuran todos los registros para el funcionamiento del microcontrolador como son definir los 218 
puertos de entrada y salida, manejo de interrupciones y configuración del envío y recepción de datos.

Luego se procede a inicializar el USB llamando a la función initUSB y se espera hasta que la enumeración del dispositivo se complete.

Ahora el procesador ingresa a un bucle en el cual realiza dos cosas, primero esperar un dato desde la computadora y si no lo recibe se da un tiempo para que pueda producirse una interrupción del teclado o del puerto serial; si no se ha producido ninguno, vuelve a esperar datos desde la computadora, quedando en un bucle infinito

La otra parte de este software es un firmware de libre uso que provee Microchip y de libre distribución que maneja la interfaz de bajo nivel entre sus dispositivos PIC16C765/45 y el Host como una base para los diseñadores de proyectos en donde se necesite una conexión USB.

Este firmware esta dividido en cuatro archivos que al momento de compilarlos junto con nuestro programa principal forma todo el software necesario para el funcionamiento de nuestra aplicación.

\section{Construcción del dispositivo}

Para el diseño del circuito esquemático se utilizo Client 99se, el cual es un sistema EDA, para diseño electrónico desarrollado por la firma PROTEL. Como todos los sistemas EDA modernos, es una herramienta integradora. Client 99se introduce ideas innovadoras que facilitan la edición rápida y eficiente de esquemas y circuitos impresos, la simulación de circuitos.

Una vez terminado el diseño esquemático, se revisaron las reglas de diseño, utilizando la correspondiente herramienta de chequeo. Al tener todo correcto, se procedió a la generación del "netlist", para la edición del circuito impreso. 
Una vez validado este, se procede al posicionado de los componentes. El posicionado es la base de un diseño final exitoso del PCB; de este dependerán muchos factores de calidad, dentro de los cuales están la calidad del ruteo y la legibilidad de los componentes en la tarjeta para su montaje fácil.

Por último, se procedió al ruteo de la tarjeta, el cual puede ser manual o automático; lo más común es combinar ambas técnicas. Este paso también involucra una serie de cuidados especiales que están relacionados con la estética del diseño como es la forma de la tarjeta, la disposición de los elementos y la tecnología de fabricación disponible.

El hardware del sistema del Braille Electrónico tiene dos terminales de conexión con la PC, estas son:

Conexión serie

Conexión USB

Queda a criterio del usuario y en función del puerto de comunicación que disponga la PC, para que el usuario escoja y configure el medio de comunicación PC-dispositivo.

El sistema mecánico dispone de dos signos generadores, un grande el cual esta escala 20:1 del signo generador normal. El mecanismo de accionamiento que se utiliza para el signo generador grande son las solenoides, mientras que para el signo generador pequeño se diseñó un mecanismo a partir del accionamiento de las solenoides del signo generador grande. (Figura 6).

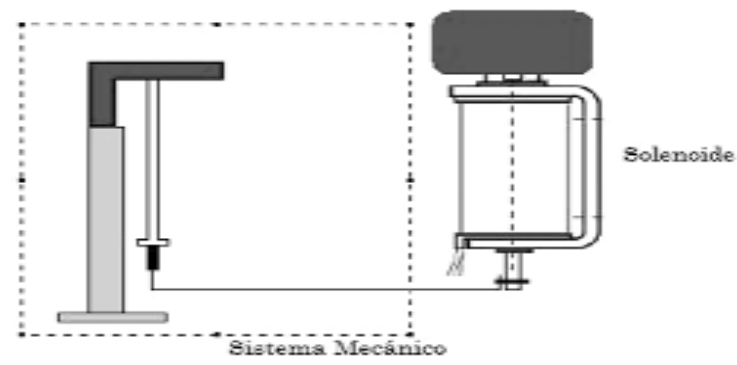

Figura 6. Estructura del Interfaz del programa del Braille Electrónico. 
Para probar Braille Electrónico se lo conecta a la computadora mediante un cable USB o serial. En la PC deberá estar ya instalado el software que se diseñó y se procede a comprobar el funcionamiento correcto del sistema.

Cada vez que enviemos un dato desde el computador, Braille Electrónico lo procesara y podrá observarse el equivalente braille de la letra que enviamos en los leds; además, la parte mecánica deberá emularlo correctamente.

\section{7.- Componentes del Sistema Braille Electrónico}

1. Dispositivo Braille Electrónico.

2. Cable de interfase RS-232 para conectar a una PC estándar al puerto serial.

3. Cable de interfase USB para conectar a una PC estándar al puerto USB.

4. Software del Braille Electrónico.

5. Fuente de alimentación de $12 \mathrm{Vcd}$.

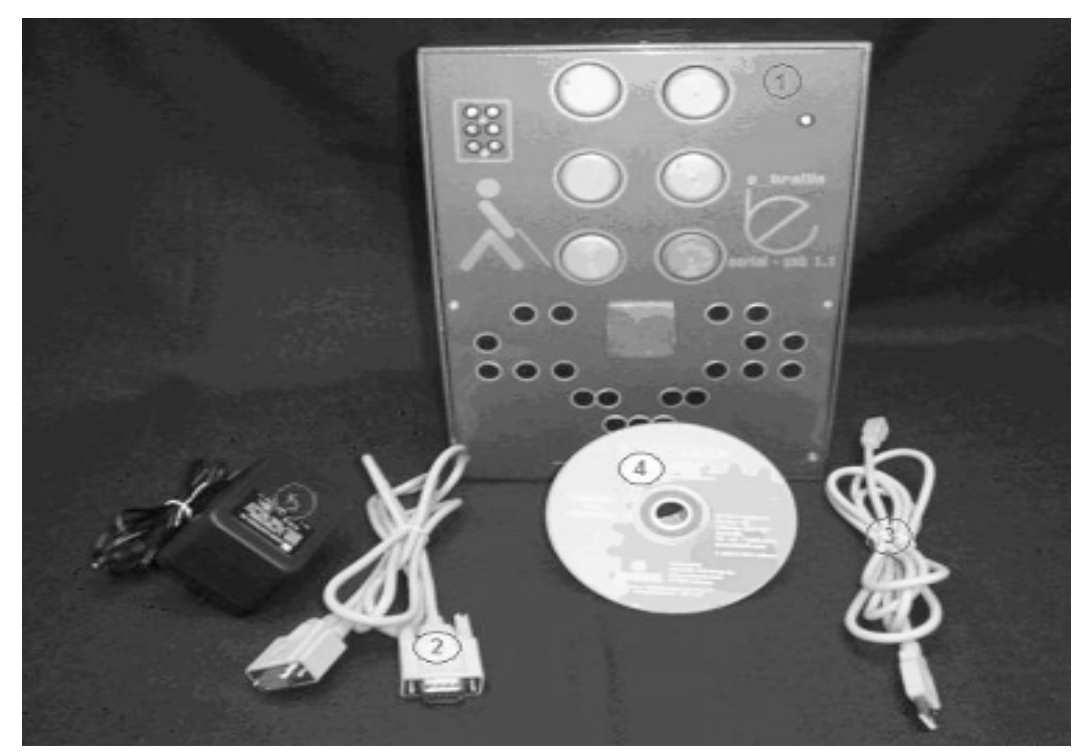

Figura 7. Componentes de Braille Electrónico 


\section{Pruebas del proyecto}

Antes de comenzar a utilizar Braille Electrónico, con una persona no vidente, el tutor deberá instalar el software en la computadora.

El kit de enseñanza de Braille Electrónico cuenta con un $C D$ auto ejecutable el cual al ingresarlo a una unidad de CDRom, lanzará la interfase mostrada en la figura 8.

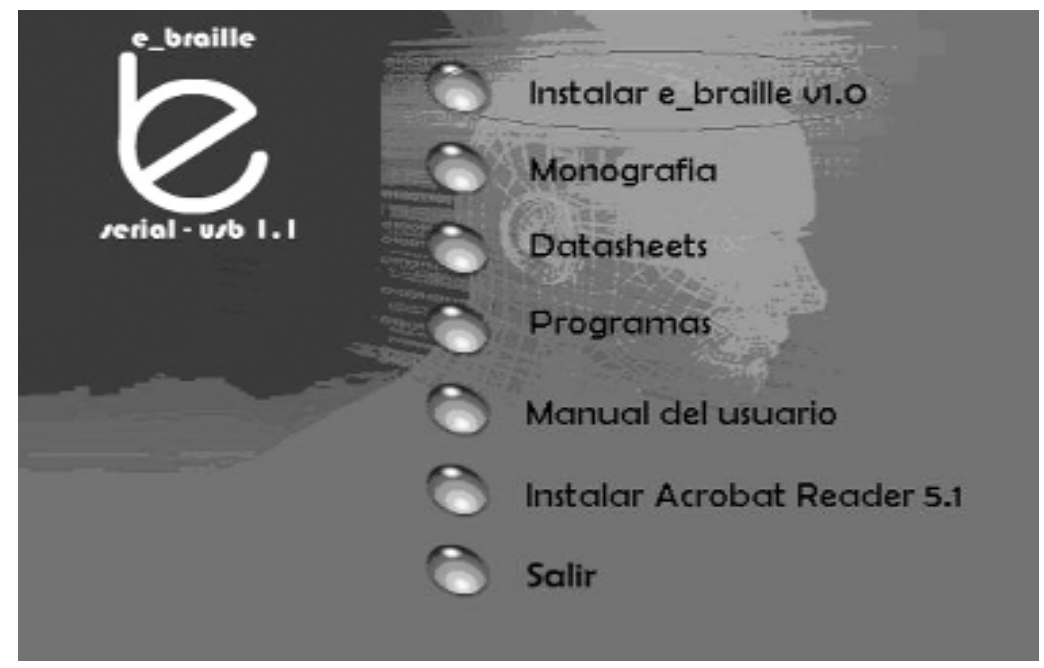

Figura 8. Interfaz del CD de Braille Electrónico

Al dar un clic sobre el botón de instalar se ejecutara el programa que carga todos los archivos necesarios para que el software de Braille Electrónico pueda funcionar en cualquier computador.

Luego se escoge el tipo de conexión que desea utilizar, la cual puede ser de tipo serial para computadoras antiguas como la conexión USB para computadores más modernos, encender el dispositivo, configurar algunos campos como son la habilitación del sonido, lectura y escritura; además deberá configurar el tipo de voz que más se acomode a las necesidades del usuario. 
También se deberá comprobar el correcto funcionamiento del sistema mecánico, es decir al tutor le corresponderá enviar algunos caracteres y comprobar que estén correctamente relacionados los enviados con los que se muestran en el panel de Braille Electrónico.

Es necesario generar instancias que basadas, en el diseño de ambientes didácticos, favorezcan el proceso de aprendizaje de las personas con limitación visual con relación al aprendizaje braille tradicional.

Los resultados que se obtuvieron con el desarrollo de esta tesis fueron satisfactorios ya que se cumplieron los objetivos planteados inicialmente para la confirmación de la hipótesis; además se pudo mejorar las características de Braille Electrónico que se trazaron con la formulación del proyecto, convirtiéndolo en un sistema de software y hardware más amigable para el usuario.

Braille Electrónico ha demostrado ser un sistema ideal para las personas totalmente ciegas que deseen acceder al aprendizaje del Sistema Braille. Este dispositivo envía la información contenida en la pantalla hasta el usuario utilizando caracteres braille dispuestos en una línea; para comunicar con el operador, cuentan con un teclado propio mediante el cual se pueden realizar todas las funciones de lectura e identificación de contenidos de la visualización.

También puede ser usado por personas videntes que deseen adquirir cierto conocimiento sobre el sistema de lectura braille, como cultura general o para convertirse en un tutor de este método.

\section{Conclusiones}

Con la realización de este proyecto podemos concluir que se ha desarrollado de manera satisfactoria una herramienta multimedia para personas no videntes, que ayuda en el aprendizaje del sistema braille además de que lo 
puede utilizar cualquier persona vidente que le interese aprender braille.

También pudimos comprobar que, utilizando el braille electrónico, la enseñanza del braille se alcanza en menor tiempo que con métodos tradicionales ayudando además, de una manera psicológica, a la persona no vidente que la está utilizando por el simple hecho de realizar una tarea que antes le era muy difícil, interactuar con una computadora.

Los autores recomendamos que se siga con un programa para la enseñanza en nuestro sistema, es decir, se empezará con la ayuda de un tutor aprendiendo primero a reconocer las letras, números y símbolos en braille en el signo grande, ayudándose de la opción de activar o desactivar la pronunciación; luego podrá realizar el test que viene en el software usando ya los controles del equipo para luego realizar lecturas independientemente del tutor y así utilizar el signo pequeño para alcanzar velocidades de lectura cada vez mayores.

A lo largo de este trabajo, se encontró que el desarrollo de software accesible para la población con limitación visual, está muy inexplorada, debido a que los desarrolladores de aplicaciones multimedia desconocen las necesidades existentes para las comunidades y al cual ellos podrían aportar sus conocimientos desde su campo de acción.

Los creadores de proyectos, al momento de diseñarlos, deben hacerlo reconociendo la necesidad de la persona que va a usar los diferentes dispositivos y no como se acostumbra a realizarlos aplicando todo el conocimiento adquirido lo que finaliza en un proyecto con demasiada tecnología y pocas prestaciones para el usuario.

Este no ha sido el caso de Braille Electrónico puesto que se ha realizado un gran esfuerzo, conjuntamente con usuarios potenciales del dispositivo, para reconocer necesidades y agregar características que hacen que este proyecto sea de gran ayuda para aplicaciones de enseñanza del sistema braille.

224 
Este trabajo servirá para adelantar futuras investigaciones, no solo en el campo del desarrollo del software sino también el área de pedagogía sobre la enseñanza del braille mediante el uso de un computador.

En cuanto al cuidado del equipo recomendamos utilizarlo con los accesorios que vienen con el sistema, es decir, no utilizar un adaptador de alimentación diferente ya que causaría daños al equipo, tampoco es recomendable usar otros tipos de cables para la conexión con el computador porque podrían dañar al PC.

No utilizar aceites ni grasas para lubricar las partes móviles ya que estas podrían dañar la tarjeta electrónica causando la destrucción del equipo.

Para finalizar, solo nos resta recordarles a padres y maestros regulares y de apoyo, que la enseñanza del Sistema de Lectoescritura Braille debe formar parte integral de la formación de niños y niñas con limitación visual. Este acercamiento hacia ellos, debe estar lleno de comprensión y respeto como personas y futuros ciudadanos, pero, sobre todo, como niños y niñas con intereses y necesidades, capaces de tomar decisiones propias.

"El acceso a la comunicación en su sentido más amplio es el acceso al conocimiento y eso es de importancia vital para nosotros si no queremos continuar siendo despreciados o protegidos por personas videntes compasivas.

No necesitamos piedad ni que nos recuerden que somos vulnerables. Tenemos que ser tratados como iguales y la comunicación es el medio por el que podemos conseguirlo".

\section{Louis Braille}


